\title{
Coding Schemes for Discrete Memoryless Multicast Networks with Rate-limited Feedback
}

\author{
Youlong $\mathrm{Wu}$ \\ Lehrstuhl für Nachrichtentechnik \\ Technische Universität München, Germany \\ youlong.wu@tum.de
}

\begin{abstract}
We propose two coding schemes for discrete memoryless multicast networks with rate-limited feedback from receivers and relays to the transmitter. The coding schemes are based on block Markov coding, joint backward decoding and hybrid relaying strategy. In each block, the receivers and relays compress their outputs and send the compression indices to the transmitter through the feedback links. In the next block, after obtaining compression indices, the transmitter sends them together with the source message. Each receiver uses backward decoding to jointly decode the source message and all compression indices. It is shown that our coding schemes strictly improve on noisy network coding and generalize Gabbai and Bross's results for the single relay channel with partial feedback, where they proposed schemes based on restricted decoding and deterministic partitioning. Motivated by our feedback schemes, we propose a new coding scheme for discrete memoryless multicast networks without feedback.
\end{abstract}

\section{INTRODUCTION}

The relay channel, first introduced by van de Meulen [1], describes a 3-node communication channel where the transmitter sends a message to the receiver with the assistance of relay. Cover and El Gamal [2] proposed two basic coding strategies: compress-forward and decode-forward, for the discrete memoryless relay channel. Both strategies are based on block Markov coding. In the compress-forward strategy, the relay compresses its outputs and sends compression index to the receiver. In the decode-forward strategy, the relay first decodes the full or part of source message and then sends the decoded message to the receiver. Both strategies have been generalized to multiple-relay channels [3]. The compressforward was later extended to a more general network-discrete memoryless networks [4], [5], [6], called noisy network coding (NNC). Recently, a distributed decode-forward coding (DDF) scheme was proposed for multicast [7] and broadcast relay networks [8], which uses the partial decode-forward at the relays and backward coding at the transmitter.

Perfect feedback from the receiver to the relay makes the relay channel is physically degraded [2], and therefore decodeforward achieves the capacity. For the case with feedback from the receiver or relay to the transmitter, the capacity is unknown in general. In [9] Gabbai and Bross studied this problem and proposed inner bounds by using restricted decoding and deterministic partitioning [10].

In this paper, we consider the general discrete memoryless multicast network with rate-limited feedback. This network consists of $N \geq 3$ nodes where the transmitter sends a message to different receivers with the assistance of multiple relays and in the presence of rate-limited feedback from the receivers and relays to the transmitter. We propose two coding schemes based on block Markov coding, joint backward decoding and hybrid relaying strategy. In our first scheme, relays and receivers use compress-forward to create their compression indices and then send them both into the forward communication and feedback channels. The transmitter, after obtaining the compression indices through feedback, sends them together with the source message. Receivers jointly decode the source message and all compression indices. Our second scheme is similar, except that the relay not only uses compress-forward as in the first scheme, but also use partial decode-forward [2] to decode the source message.

Our strategy is reminiscent of the noisy network coding for general networks [5], [6] in the sense the relays and receivers compress their channel outputs and send these compression indices over the feedback links. However, our schemes have the transmitter forward the receivers and relays' compression messages, instead of creating a new compression message. This is similar to the schemes in [11] for the broadcast channel, where the transmitter forwards the receivers' compression messages. It is shown that our coding schemes generalize Gabbai and Bross's results [9] for the relay channel with relaytransmitter feedback. For some channels, such as the Gaussian relay channel and $Z$ relay channels, our coding schemes improve over the NNC scheme [5], the DDF coding scheme [7], [8] and all known lower bounds on the achievable rate in the absence of feedback. Motivated by our feedback schemes, we propose a new coding scheme for discrete memoryless multicast networks without feedback.

Notation: We use capital letters to denote random variables and small letters for their realizations, e.g. $X$ and $x$. For $j \in$ $\mathbb{Z}^{+}$, let $X_{1}^{j}:=\left(X_{1}, \ldots, X_{j}\right)$ and $X_{2}^{j}:=\left(X_{2}, \ldots, X_{j}\right)$. Given a finite set $\mathcal{X}$, we denote by $|\mathcal{X}|$ its cardinality. Given a set $\mathcal{A} \subseteq$ $[2: N]$, and let $\mathcal{A}^{c}:=[2: N] \backslash \mathcal{A}$. A tuple of random variables is denoted as $X(\mathcal{A}):=\left[X_{k}: k \in \mathcal{A}\right]$. For $n \in \mathbb{Z}^{+}$, let $\mathbf{1}_{[n]}$ denote the all-one tuple of length $n$, e.g., $\mathbf{1}_{[3]}=(1,1,1)$.

\section{SYSTEM MODEL}

Consider an $N$-node discrete memoryless (DM) multicast networks with feedback from receivers and relays to the 
transmitter, see Figure 1. Let $\mathcal{R}$ and $\mathcal{D}$ denote the set of relays and receivers, respectively, where $\mathcal{R} \subset[2: N]$ and $\mathcal{D}=$ $[2: N] \backslash \mathcal{R}$. This setup is characterized by $2 N$ finite alphabets $\mathcal{X}_{1}, \ldots, \mathcal{X}_{N}, \mathcal{Y}_{1}, \ldots, \mathcal{Y}_{N}$, a channel law $P_{Y_{1} \ldots Y_{N} \mid X_{1}, \ldots, X_{N}}$ and nonnegative feedback rates $R_{\mathrm{Fb}, k}$, for $k \in[2: N]$. Specifically, at discrete-time $i$, node $j \in[1: N]$ sends input $x_{j, i} \in \mathcal{X}_{j}$ and then observes output $y_{j, i} \in \mathcal{Y}_{j}$. After observing $Y_{k, i}$, for $k \in[2: N]$, node $k$ sends a feedback signal $F_{k, i} \in \mathcal{F}_{k, i}$ to the transmitter, where $\mathcal{F}_{k, i}$ denotes the finite alphabet of $F_{k, i}$. The feedback link between the transmitter and node $k$ is assumed to be instantaneous, noiseless and rate-limited to $R_{\mathrm{Fb}, k}$ bits on average. In other words, if the transmission takes place over a total blocklength $n$, then

$$
\left|\mathcal{F}_{k, 1}\right| \times \cdots \times\left|\mathcal{F}_{k, n}\right| \leq 2^{n R_{\mathrm{Fb}, k}}, \quad k \in[2: N] .
$$

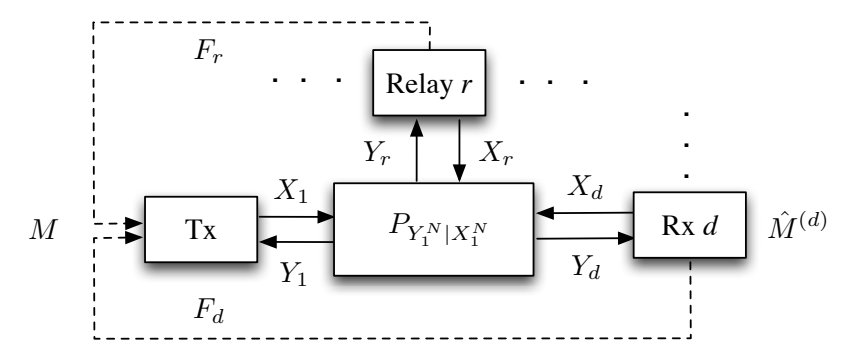

Fig. 1. $N$-node discrete memoryless multicast network with partial feedback

In the transmission, the transmitter communicates a message $M \in\left[1: 2^{n R}\right]$ to the set of receivers $\mathcal{D}$ with the assistance of the relays $\mathcal{R}$. A $\left(2^{n R}, n\right)$ code for this channel has

- a message set $\left[1: 2^{n R}\right]$,

- a source encoder that maps $\left(M, Y_{1}^{i-1}, F_{2}^{i-1}, \ldots, F_{N}^{i-1}\right)$ to the channel input $X_{1, i}\left(M, Y_{1}^{i-1}, F_{2}^{i-1}, \ldots, F_{N}^{i-1}\right)$, for each time $i \in[1: n]$,

- a set of relay and receiver encoders that maps $Y_{k}^{i-1}$ to a sequence $X_{k, i}\left(Y_{k}^{i-1}\right)$, for $k \in[2: N]$ and $i \in[1: n]$,

- a set of feedback-encoder that is to produce feedback symbols $F_{k, i}\left(Y_{k, 1}^{i}\right)$, for $k \in[2: N]$ and $i \in[1: n]$,

- a set of decoders that estimates $\hat{M}^{(d)}$ based on $Y_{d}^{n}$, for $d \in \mathcal{D}$.

Suppose $M$ is uniformly distributed over the message set. A rate $R$ is called achievable if for every blocklength $n$, there exists a $\left(2^{n R}, n\right)$ code such that the average probability of error $P_{e}^{(n)}=\operatorname{Pr}\left[\hat{M}^{(d)} \neq M\right.$, for some $\left.d \in \mathcal{D}\right]$ tends to 0 as the $n$ tends to infinity. The capacity is the supremum of the set of achievable rates $R$ such that $\lim _{n \rightarrow \infty} P_{e}^{(n)}=0$.

\section{MAin Results}

This section presents our main results. The proofs are given in Sections IV and V.

Theorem 1. For DM multicast networks with feedback from the receivers and relays to the transmitter, the rate $R$ is achievable if

$$
\begin{aligned}
R \leq I\left(X_{1}, X(\mathcal{T}) ; \hat{Y}\left(\mathcal{T}^{c}\right), Y_{d} \mid X\left(\mathcal{T}^{c}\right)\right) \\
-I\left(\hat{Y}(\mathcal{T}) ; Y(\mathcal{T}) \mid X_{1}^{N}, Y_{d}, \hat{Y}\left(\mathcal{T}^{c}\right)\right)
\end{aligned}
$$

for all $d \in \mathcal{D}, \mathcal{T} \subset[2: N]$ with $\mathcal{T}^{c} \cap \mathcal{D} \neq \emptyset$ and for some pmf

$$
\left[\prod_{k=2}^{N} P_{X_{k}}\right] P_{X_{1} \mid X_{2}^{N}} P_{Y_{1}^{N} \mid X_{1}^{N}}\left[\prod_{k=2}^{N} P_{\hat{Y}_{k} \mid X_{k} Y_{k}}\right]
$$

such that

$$
R_{F b, k} \geq I\left(\hat{Y}_{k} ; Y_{k} \mid X_{k}\right), \quad \text { for } k \in[2: N] .
$$

Proof: See Section IV-A.

Remark 1. Comparing the lower bound in Theorem 1 with the NNC lower bound [5, Theorem 1], our rates includes the NNC lower bound if feedback rates are sufficient large such that (4) holds for all pmfs (3), since in (3) we allow the joint distribution $\prod_{k=2}^{N} P_{X_{k}} P_{X_{1} \mid X_{2}^{N}}$ instead of $\prod_{k=1}^{N} P_{X_{k}}$.

Theorem 2. For DM multicast networks with feedback from the receivers and relays to the transmitter, the rate $R$ is achievable if

$$
\begin{gathered}
R \leq I\left(X_{1} ; \hat{Y}_{2}^{N}, Y_{d} \mid U_{2}^{N}, X_{2}^{N}\right)+\min _{r \in \mathcal{R}} I\left(U_{r} ; Y_{r} \mid X_{r}\right) \\
R \leq I\left(X_{1}, X(\mathcal{T}), U(\mathcal{T}) ; \hat{Y}\left(\mathcal{T}^{c}\right), Y_{d} \mid X\left(\mathcal{T}^{c}\right), U\left(\mathcal{T}^{c}\right)\right) \\
\quad-I\left(\hat{Y}(\mathcal{T}) ; Y(\mathcal{T}) \mid U_{2}^{N}, X_{1}^{N}, \hat{Y}\left(\mathcal{T}^{c}\right), Y_{d}\right)
\end{gathered}
$$

for all $d \in \mathcal{D}, \mathcal{T} \subset[2: N]$ with $\mathcal{T}^{c} \cap \mathcal{D} \neq \emptyset$, and for some pmf

$$
\begin{aligned}
& {\left[\prod_{k=2}^{N} P_{X_{k} U_{k}}\right] P_{X_{1} \mid X_{2}^{N} U_{2}^{N}} P_{Y_{1}^{N} \mid X_{1}^{N}}} \\
& \quad \times\left[\prod_{r \in \mathcal{R}} P_{\hat{Y}_{r} \mid U_{r} X_{r} Y_{r}}\right]\left[\prod_{d \in \mathcal{D}} P_{\hat{Y}_{d} \mid X_{d} Y_{d}}\right]
\end{aligned}
$$

such that

$$
\begin{aligned}
& R_{F b, r} \geq\left(\hat{Y}_{r} ; Y_{r} \mid X_{r}, U_{r}\right), \quad \text { for } r \in \mathcal{R} \\
& R_{F b, d} \geq I\left(\hat{Y}_{d} ; Y_{d} \mid X_{d}\right), \quad \text { for } d \in \mathcal{D} .
\end{aligned}
$$

Proof: See Section IV-B

Remark 2. By setting $U_{2}=\cdots=U_{N}=$ const., the achievable rate in Theorem 2 specializes to the rate in Theorem 1. (Setting $U_{2}=\cdots=U_{N}=$ const. means that all relay nodes only perform compress-forward without partially decode-forwarding the source message.)

Based on coding schemes for Theorems 1 and 2, we propose another coding scheme for DM multicast networks without feedback. The new achievable rate is shown below.

Theorem 3. For DM multicast networks without feedback, the rate $R$ is achievable if (8) holds for all $d \in \mathcal{D}, \mathcal{T} \subset[2: N]$ with $\mathcal{T}^{c} \cap \mathcal{D} \neq \emptyset$, and for some pmf

$$
\begin{aligned}
& {\left[\prod_{k=2}^{N} P_{V_{k}} P_{X_{k} \mid V_{k}} P_{U_{k} \mid V_{k}}\right] P_{X_{1} \mid V_{2}^{N} U_{2}^{N}}} \\
& \quad \times P_{Y_{1}^{N} \mid X_{1}^{N}}\left[\prod_{r \in \mathcal{R}} P_{\hat{Y}_{r} \mid U_{r} V_{r} X_{r} Y_{r}}\right]\left[\prod_{d \in \mathcal{D}} P_{\hat{Y}_{d} \mid V_{d} X_{d} Y_{d}}\right]
\end{aligned}
$$




$$
\begin{aligned}
& R \leq I\left(X_{1} ; \hat{Y}_{2}^{N}, Y_{d} \mid U_{2}^{N}, V_{2}^{N}, X_{2}^{N}\right)+\min _{r \in \mathcal{R}} I\left(U_{r} ; Y_{r} \mid V_{r}, X_{r}\right) \\
& R \leq I\left(X_{1}, X(\mathcal{T}), U(\mathcal{T}), V(\mathcal{T}) ; \hat{Y}\left(\mathcal{T}^{c}\right), Y_{d} \mid U\left(\mathcal{T}^{c}\right), V\left(\mathcal{T}^{c}\right), X\left(\mathcal{T}^{c}\right)\right)-I\left(\hat{Y}(\mathcal{T}) ; Y(\mathcal{T}) \mid U_{2}^{N}, V_{2}^{N}, X_{1}^{N}, \hat{Y}\left(\mathcal{T}^{c}\right), Y_{d}\right)
\end{aligned}
$$

such that

$$
\begin{aligned}
& \sum_{r \in \mathcal{T} \cap \mathcal{R}} I\left(\hat{Y}_{r} ; Y_{r} \mid U_{r}, V_{r}, X_{r}\right)+\sum_{d \in \mathcal{T} \cap \mathcal{D}} I\left(\hat{Y}_{d} ; Y_{d} \mid V_{d}, X_{d}\right) \\
& \leq I\left(X(\mathcal{T}) ; Y_{1} \mid U_{2}^{N}, V_{2}^{N}, X\left(T^{c}\right), X_{1}\right) .
\end{aligned}
$$

Example 1. (The relay channel with relay-transmitter feedback) Consider the relay channel with perfect feedback from the relay to the transmitter, see Figure 2.

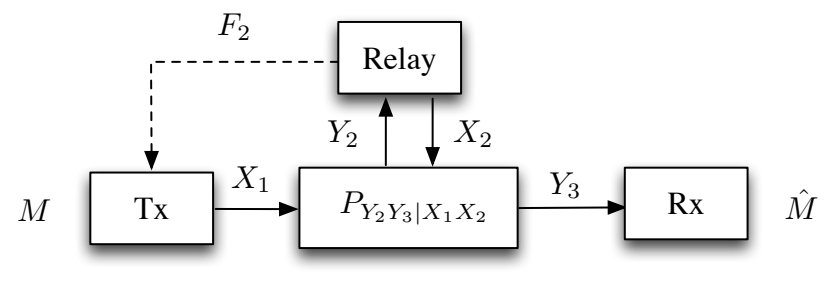

Fig. 2. Relay channel with relay-transmitter feedback

Let $\hat{Y}_{3}=\emptyset$, then Theorem 1 specializes to

$$
\begin{aligned}
& R \leq I\left(X_{1} ; \hat{Y}_{2}, Y_{3} \mid X_{2}\right) \\
& \left.R \leq I\left(X_{1}, X_{2} ; Y_{3}\right)-I\left(\hat{Y}_{2} ; Y_{2} \mid X_{1}, X_{2}, Y_{3}\right)\right\}
\end{aligned}
$$

for some pmf $P_{X_{1} X_{2}} P_{\hat{Y}_{2} \mid X_{2} Y_{2}}$. Let $U_{3}=\hat{Y}_{3}=\emptyset$, then Theorem 2 specializes to

$$
\begin{aligned}
& R \leq I\left(X_{1} ; \hat{Y}_{2}, Y_{3} \mid U_{2}, X_{2}\right)+I\left(U_{2} ; Y_{2} \mid X_{2}\right) \\
& R \leq I\left(X_{1}, X_{2} ; Y_{3}\right)-I\left(\hat{Y}_{2} ; Y_{2} \mid U_{2}, X_{1}, X_{2}, Y_{3}\right)
\end{aligned}
$$

for some pmf $P_{X_{1} X_{2} U_{2}} P_{\hat{Y}_{2} \mid X_{2} U_{2} Y_{2}}$.

In [9] Gabbai and Bross studied this channel and proposed coding schemes based on restricted decoding and deterministic partitioning. The rates (11) and (12) recover Gabbai and Bross's rates of Theorems 2 and 3 in [9], respectively.

By using NNC [5], the rate $R$ satisfying

$$
\begin{aligned}
& R \leq I\left(X_{1} ; \hat{Y}_{2}, Y_{3} \mid X_{2}\right) \\
& \left.R \leq I\left(X_{1}, X_{2} ; Y_{3}\right)-I\left(\hat{Y}_{2} ; Y_{2} \mid X_{1}, X_{2}, Y_{3}\right)\right\}
\end{aligned}
$$

is achievable for any pmf $P_{X_{1}} P_{X_{2}} P_{\hat{Y}_{2} \mid X_{2} Y_{2}}$, which coincides with the compress-forward lower bound [2, Theorem 6].

By using DDF [7], [8], the rate $R$ satisfying

$$
\begin{aligned}
& R \leq I\left(X_{1}, X_{2} ; Y_{3}\right) \\
& R \leq I\left(U_{2} ; Y_{2} \mid X_{2}\right)+I\left(X_{1} ; Y_{3} \mid X_{2}, U_{2}\right)
\end{aligned}
$$

is achievable for any pmf $P_{X_{1} X_{2} U_{2}}$, which coincides with the partial decode-forward lower bound [2, Theorem 7].

The lower bound (12) includes (13) and (14). In [9] Gabbai and Bross showed that for the Gaussian and $Z$ relay channels, the lower bound (12) improves on the known lower bounds on the achievable rate in the absence of feedback, including the compress-forward lower bound in (13), and the partial decodeforward lower bound in (14). In view of this fact, we have the following corollary:

Corollary 1. For the DM single-relay channel with relaytransmitter feedback, our coding schemes recover Gabbai and Bross's results, and can strictly improve on NNC [5], DDF [7] and all known lower bounds on the achievable rate in the absence of feedback.

\section{ACHIEVAble Rates FOR DM MUlticast Networks WITH FEEDBACK}

\section{A. Scheme $1 A$}

In this subsection we present a block Markov coding scheme where a sequence of $B$ i.i.d message $m_{b}, b \in[1: B]$ is sent over $B+1$ block. In each block $b \in[1: B+1]$, Relay $r \in \mathcal{R}$ uses compress-forward to compress its observed outputs $Y_{k, b}^{n}$, and then send the compression index into the feedback link. After obtaining all compression indices through feedback, the transmitter sends them together with the source message in the next block. Define $\mathbf{1}_{b-1}:=\left(l_{2, b-1}, \ldots, l_{N, b-1}\right)$ and $\hat{\mathbf{l}}_{b-1}:=$ $\left(\hat{l}_{2, b-1}, \ldots, \hat{l}_{N, b-1}\right)$, for $b \in[1: B+1]$. Let $\mathbf{l}_{0}=\mathbf{1}_{[N-1]}$ and $m_{B+1}=1$.

1) Codebooks: Fix the pmf in (3). For each block $b \in$ $[1: B+1]$ and $k \in[2: N]$, randomly and independently generate $2^{n \hat{R}_{k}}$ sequences $x_{k, b}^{n}\left(l_{k, b-1}\right) \sim \prod_{i=1}^{n} P_{X_{k}}\left(x_{k, b, i}\right)$, $l_{k, b-1} \in\left[1: 2^{n \hat{R}_{k}}\right]$. For each $l_{k, b-1}$, randomly and independently generate $2^{n \hat{R}_{k}}$ sequences $\hat{y}_{k, b}^{n}\left(l_{k, b} \mid l_{k, b-1}\right) \sim$ $\prod_{i=1}^{n} P_{\hat{Y}_{k} \mid X_{k}}\left(\hat{y}_{k, b, i} \mid x_{k, b, i}\right)$. For each $\mathbf{l}_{b-1}$, randomly and independently generate $2^{n R}$ sequences $x_{1, b}^{n}\left(m_{b} \mid \mathbf{l}_{b-1}\right) \sim$ $\prod_{i=1}^{n} P_{X_{1} \mid X_{2}^{N}}\left(x_{1, b, i} \mid x_{2, b, i}, \ldots, x_{N, b, i}\right), m_{b} \in\left[1: 2^{n R}\right]$.

2) Source encoding: In each block $b \in[1: B+1]$, assume that the transmitter already knows $\mathbf{I}_{b-1}$ through feedback links. It sends $x_{1, b}^{n}\left(m_{b} \mid \mathbf{l}_{b-1}\right)$.

3) Relay and receiver encoding: Relays and receivers both use compress-forward . In each block $b \in[1: B]$, node $k \in$ $[2: N]$ compresses $y_{k, b}^{n}$ by finding a unique index $l_{k, b}$ such that

$$
\left(x_{k, b}^{n}\left(l_{k, b-1}\right), \hat{y}_{k, b}^{n}\left(l_{k, b} \mid l_{k, b-1}\right), y_{k, b}^{n}\right) \in \mathcal{T}_{\epsilon}^{n}\left(P_{X_{k} Y_{k} \hat{Y}_{k}}\right) .
$$

Then, it sends $l_{k, b}$ through the feedback link at rate $\hat{R}_{k} \leq$ $R_{\mathrm{Fb}, k}$ and in block $b+1$ sends $x_{k, b+1}^{n}\left(l_{k, b}\right)$.

4) Decoding: Receivers perform joint backward decoding. For each block $b \in[B+1, \ldots, 1]$, Receiver $d \in \mathcal{D}$ looks for 
$\left(\hat{m}_{b}, \hat{\mathbf{l}}_{b-1}\right)$ such that ${ }^{1}$

$$
\begin{aligned}
& \left(x_{1, b}^{n}\left(\hat{m}_{b} \mid \hat{\mathbf{l}}_{b-1}\right), x_{2, b}^{n}\left(\hat{l}_{2, b-1}\right), \ldots, x_{N, b}^{n}\left(\hat{l}_{N, b-1}\right), y_{d, b}^{n},\right. \\
& \left.\quad \hat{y}_{2, b}^{n}\left(\hat{l}_{2, b} \mid \hat{l}_{2, b-1}\right), \ldots, \hat{y}_{N, b}^{n}\left(\hat{l}_{N, b} \mid \hat{l}_{N, b-1}\right)\right) \in \mathcal{T}_{\epsilon}^{n}\left(P_{X_{1}^{N} \hat{Y}_{2}^{N} Y_{d}}\right) .
\end{aligned}
$$

5) Analysis: See [12] for details.

\section{B. Scheme $1 B$}

Note that in Scheme 1A above, the relays and receivers only perform compress-forward . In this subsection, we present a scheme where relays perform mixed compress-forward and partial decode-forward.

1) Codebooks: Fix the pmf in (6). Transmission takes place in $B+1$ blocks each consisting of $n$ transmissions. For block $b \in[1: B]$, split the message $m_{b}$ into $\left(m_{b}^{\prime}, m_{b}^{\prime \prime}\right)$, where $m_{b}^{\prime}$ and $m_{b}^{\prime \prime}$ are independently and uniformly distributed over the set $\left[1: 2^{n R^{\prime}}\right]$ and $\left[1: 2^{n R^{\prime \prime}}\right]$, respectively, where $R^{\prime}, R^{\prime \prime} \geq 0$ and so that $R=R^{\prime}+R^{\prime \prime}$. Let $m_{B+1}^{\prime \prime}=m_{B+1}^{\prime}=1$.

- For each $r \in \mathcal{R}$ and block $b \in[1: B+1]$, randomly and independently generate $\left.2^{n\left(R^{\prime}+\hat{R}_{r}\right.}\right)$ sequences $x_{r, b}^{n}\left(m_{b-1}^{\prime}, l_{r, b-1}\right) \sim \prod_{i=1}^{n} P_{X_{r}}\left(x_{r, b, i}\right)$, with $m_{b-1}^{\prime} \in[1$ : $\left.2^{n R^{\prime}}\right]$ and $l_{r, b-1} \in\left[1: 2^{n \hat{R}_{r}}\right]$. For each $\left(m_{b-1}^{\prime}, l_{r, b-1}\right)$, randomly and independently generate $2^{n R^{\prime}}$ sequences $u_{r, b}^{n}\left(m_{b}^{\prime} \mid m_{b-1}^{\prime}, l_{r, b-1}\right) \sim \prod_{i=1}^{n} P_{U_{r} \mid X_{r}}\left(u_{r, b, i} \mid x_{r, b, i}\right)$. For each $\left(m_{b}^{\prime}, m_{b-1}^{\prime}, l_{r, b-1}\right)$, randomly and independently generate $2^{n \hat{R}_{r}}$ sequences $\hat{y}_{r, b}^{n}\left(l_{r, b} \mid m_{b}^{\prime}, m_{b-1}^{\prime}, l_{r, b-1}\right) \sim$ $\prod_{i=1}^{n} P_{\hat{Y}_{r} \mid U_{r} X_{r}}\left(\hat{y}_{r, b, i} \mid u_{r, b, i}, x_{r, b, i}\right)$.

- For each $d \in \mathcal{D}$ and block $b \in[1: B+1]$, randomly and independently generate $2^{n \hat{R}_{d}}$ sequences $x_{d, b}^{n}\left(l_{d, b-1}\right) \sim \prod_{i=1}^{n} P_{X_{d}}\left(x_{d, b, i}\right), l_{d, b-1} \in\left[1: 2^{n R_{d}}\right]$. For each $l_{d, b-1}$, randomly and independently generate $2^{n R^{\prime}}$ sequences $u_{d, b}^{n}\left(m_{b}^{\prime} \mid l_{d, b-1}\right) \sim \prod_{i=1}^{n} P_{U_{d} \mid X_{d}}\left(u_{d, b, i} \mid x_{d, b, i}\right)$. Similarly, for each $l_{d, b-1}$, randomly and independently generate $2^{n \hat{R}_{d}}$ sequences $\hat{y}_{d, b}^{n}\left(l_{d, b} \mid l_{d, b-1}\right) \sim$ $\prod_{i=1}^{n} P_{\hat{Y}_{d} \mid X_{d}}\left(\hat{y}_{d, b, i} \mid x_{d, b, i}\right)$.

For each $\left(m_{b}^{\prime}, m_{b-1}^{\prime}, \mathbf{l}_{b-1}\right)$, randomly and independently generate $2^{n R^{\prime \prime}}$ sequences $x_{1, b}^{n}\left(m_{b}^{\prime \prime} \mid m_{b}^{\prime}, m_{b-1}^{\prime}, \mathbf{l}_{b-1}\right) \sim$ $\prod_{i=1}^{n} P_{X_{1} \mid U_{2}^{N} X_{2}^{N}}\left(x_{1, b, i} \mid x_{2, b, i}, u_{2, b, i}, \ldots, x_{N, b, i}, u_{N, b, i}\right)$.

2) Source encoding: In each block $b \in[1: B+1]$, assume that the transmitter already knows $\mathbf{l}_{b-1}$ through the feedback links. It sends $x_{1, b}^{n}\left(m_{b}^{\prime \prime} \mid m_{b}^{\prime}, m_{b-1}^{\prime}, \mathbf{l}_{b-1}\right)$.

3) Relay encoding: Relay nodes use hybrid compressforward and decode-forward. For each block $b \in[1: B+1]$, assume that Relay $r \in \mathcal{R}$ already knows $\hat{m}_{b-1}^{\prime}$ from block $b-1$. It looks for a unique index $\hat{m}_{b}^{\prime}$ s.t

$\left(x_{r, b}^{n}\left(\hat{m}_{b-1}^{\prime}, l_{r, b-1}\right), u_{r, b}^{n}\left(\hat{m}_{b}^{\prime} \mid \hat{m}_{b-1}^{\prime}, l_{r, b-1}\right), y_{r, b}^{n}\right) \in \mathcal{T}_{\epsilon}^{n}\left(P_{X_{r} Y_{r} U_{r}}\right)$

then it compresses $y_{r, b}^{n}$ by finding a unique index $l_{r, b}$ such that

$$
\begin{aligned}
& \left(u_{r, b}^{n}\left(\hat{m}_{b}^{\prime} \mid \hat{m}_{b-1}^{\prime}, l_{r, b-1}\right), x_{r, b}^{n}\left(\hat{m}_{b-1}^{\prime}, l_{r, b-1}\right),\right. \\
& \left.\quad \hat{y}_{r, b}^{n}\left(l_{r, b} \mid \hat{m}_{b}^{\prime}, \hat{m}_{b-1}^{\prime}, l_{r, b-1}\right), y_{r, b}^{n}\right) \in \mathcal{T}_{\epsilon}^{n}\left(P_{U_{r} X_{r} Y_{r} \hat{Y}_{r}}\right) .
\end{aligned}
$$

\footnotetext{
${ }^{1}$ Receiver $d \in \mathcal{D}$ knows $l_{d, b-1}$ since it generated this index. Since each Receiver $d$ makes its own estimate of $m_{b}$ and $\mathbf{l}_{b-1}$, the precise notation is $\left(\hat{m}_{b}^{(d)}, \hat{\mathbf{l}}_{b-1}^{(d)}\right)$. For simplicity, we omit the superscript $(d)$.
}

Then, it sends $l_{r, b}$ through the feedback link at rate $\hat{R}_{r} \leq R_{\mathrm{Fb}, r}$ and in block $b+1$ sends $x_{r, b+1}^{n}\left(\hat{m}_{b}^{\prime}, l_{r, b}\right)$.

4) Receiver encoding: Receiver $d \in \mathcal{D}$ compresses $y_{d, b}^{n}$ by finding a unique index $l_{d, b}$ such that

$$
\left(x_{d, b}^{n}\left(l_{d, b-1}\right), \hat{y}_{d, b}\left(l_{d, b} \mid l_{d, b-1}\right), y_{d, b}^{n}\right) \in \mathcal{T}_{\epsilon}^{n}\left(P_{X_{d} Y_{d} \hat{Y}_{d}}\right) .
$$

Then, it sends $l_{d, b}$ through the feedback link at rate $\hat{R}_{d} \leq$ $R_{\mathrm{Fb}, d}$ and in block $b+1$ sends $x_{d, b+1}^{n}\left(l_{d, b}\right)$.

5) Decoding: Receiver $d \in \mathcal{D}$ performs backward decoding. For each block $b \in[B+1, \ldots, 1]$, it looks for $\left(\hat{m}_{b}^{\prime \prime}, \hat{m}_{b-1}^{\prime}, \hat{\mathbf{l}}_{b-1}\right)$ such that

$$
\begin{array}{r}
\left(x_{1, b}^{n}\left(\hat{m}_{b}^{\prime \prime} \mid \hat{m}_{b}^{\prime}, \hat{m}_{b}^{\prime}, \hat{\mathbf{l}}_{b-1}\right), \mathbf{x}_{b}^{n}(\mathcal{R}), \mathbf{x}_{b}^{n}(\mathcal{D}), \mathbf{u}_{b}^{n}(\mathcal{R}), \mathbf{u}_{b}^{n}(\mathcal{D}),\right. \\
\left.\hat{\mathbf{y}}_{b}^{n}(\mathcal{R}), \hat{\mathbf{y}}_{b}^{n}(\mathcal{D}), y_{d, b}^{n}\right) \in \mathcal{T}_{\epsilon}^{n}\left(P_{X_{1}^{N} U_{2}^{N} \hat{Y}_{2}^{N} Y_{d}}\right)
\end{array}
$$

where $\mathbf{x}_{b}^{n}(\mathcal{R}):=\left[x_{r, b}^{n}\left(\hat{m}_{b-1}^{\prime}, \hat{l}_{r, b-1}\right): r \in \mathcal{R}\right], \mathbf{x}_{b}^{n}(\mathcal{D}):=$ $\left[x_{d, b}^{n}\left(\hat{l}_{d, b-1}\right): d \in \mathcal{D}\right], \mathbf{u}_{b}^{n}(\mathcal{R}):=\left[u_{r, b}^{n}\left(\hat{m}_{b}^{\prime} \mid \hat{m}_{b-1}^{\prime}, \hat{l}_{r, b-1}\right):\right.$ $r \in \mathcal{R}], \mathbf{u}_{b}^{n}(\mathcal{D}):=\left[u_{d, b}^{n}\left(\hat{m}_{b}^{\prime} \mid \hat{l}_{d, b-1}\right): d \in \mathcal{D}\right]$ and $\hat{\mathbf{y}}_{b}^{n}(\mathcal{R}):=\left[\hat{y}_{r, b}^{n}\left(\hat{l}_{r, b} \mid \hat{m}_{b}^{\prime}, \hat{m}_{b-1}^{\prime}, \hat{l}_{r, b-1}\right): r \in \mathcal{R}\right], \hat{\mathbf{y}}_{b}^{n}(\mathcal{D}):=$ $\left[\hat{y}_{d, b}^{n}\left(\hat{l}_{d, b} \mid \hat{l}_{d, b-1}\right): d \in \mathcal{D}\right]$.

6) Analysis: See [12] for details.

\section{ACHIEVAble Rates FOR DM Multicast Network}

In Section IV we proposed two block Markov coding schemes for DM multicast networks in the presence of instantaneous, rate-limited and noiseless feedback. Recall the NNC scheme [5], [6] for DM multicast networks without feedback, where each node (including the transmitter) compresses its observation and sends the new compression index in the next block. Comparing our coding scheme with NNC, we observe that both schemes involve block Markov coding, compressing channel outputs and sending compression messages. However, our schemes allow hybrid relaying strategy at relay nodes, and in each block, instead of creating new compression index, the transmitter forwards all compression indices sent by receivers and relays from the previous block. In our scheme, different nodes operate differently according to the features of the network, which leads to a larger achievable rate than NNC, as shown by the example in Section III.

Motivated by Scheme 1A and 1B, we propose another scheme for $N$-node DM multicast networks without feedback, see Figure 3. The main idea is as follows: in each block $b$, node $k \in[2: N]$ creates a compression index $l_{k, b-1}$ and sends $\left(l_{k, b-1}, l_{k, b-2}\right)$. The transmitter first decodes compression indices $\mathbf{l}_{b-1}$ based on $Y_{1, b}^{n}$, which is in essence a coding problem on a multiple access channel $P_{Y_{1} \mid X_{2}, \ldots, X_{N}}$ with side information $X_{1}$. Then in block $b+1$, the transmitter sends compression messages $\mathbf{l}_{b-1}$ and the source message $m_{b+1}$. In this section we present a scheme extending Scheme $1 \mathrm{~B}$ to DM multicast networks. Similar extensions can be applied to Scheme 1A.

1) Codebooks: Fix the pmf in (9). Transmission takes place in $B+2$ blocks each consisting of $n$ transmissions. For block $b \in[1: B]$, split the message $m_{b}$ into $\left(m_{b}^{\prime}, m_{b}^{\prime \prime}\right)$, where $m_{b}^{\prime}$ and $m_{b}^{\prime \prime}$ are independently and uniformly distributed over the 
$M$

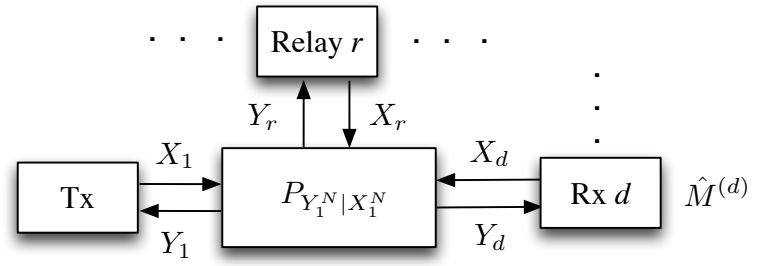

Fig. 3. Discrete memoryless multicast network without feedback

sets $\in\left[1: 2^{n R^{\prime}}\right]$ and $\left[1: 2^{n R^{\prime \prime}}\right]$, respectively, where $R^{\prime}, R^{\prime \prime} \geq$ 0 and so that $R=R^{\prime}+R^{\prime \prime}$. Let $\mathbf{l}_{-1}=\mathbf{l}_{0}=\mathbf{1}_{[N-1]}$ and $m_{B+1}^{\prime \prime}=m_{B+1}^{\prime}=m_{B+2}^{\prime \prime}=m_{B+2}^{\prime}=1$.

- For each $r \in \mathcal{R}$ and block $b \in[1: B+2]$, randomly and independently generate $2^{n\left(R^{\prime}+\hat{R}_{r}\right)}$ sequences $v_{r, b}^{n}\left(m_{b-1}^{\prime}, l_{r, b-2}\right) \sim \prod_{i=1}^{n} P_{V_{r}}\left(v_{r, b, i}\right)$, with $m_{b-1}^{\prime} \in[1$ : $\left.2^{n R^{\prime}}\right]$ and $l_{r, b-2} \in\left[1: 2^{n \hat{R}_{r}}\right]$. For each $\left(m_{b-1}^{\prime}, l_{r, b-2}\right)$, randomly and independently generate $2^{n \hat{R}_{r}}$ sequences $x_{r, b}^{n}\left(l_{r, b-1} \mid m_{b-1}^{\prime}, l_{r, b-2}\right) \sim \prod_{i=1}^{n} P_{X_{r} \mid V_{r}}\left(x_{r, b, i} \mid v_{r, b, i}\right)$, with $l_{r, b-1} \in\left[1: 2^{n \hat{R}_{r}}\right]$. For each pair $\left(m_{b-1}^{\prime}, l_{r, b-2}\right)$, randomly and independently generate $2^{n R^{\prime}}$ sequences $u_{r, b}^{n}\left(m_{b}^{\prime} \mid m_{b-1}^{\prime}, l_{r, b-2}\right) \sim \prod_{i=1}^{n} P_{U_{r} \mid V_{r}}\left(u_{r, b, i} \mid v_{r, b, i}\right)$. For each $\left(m_{b}^{\prime}, m_{b-1}^{\prime}, l_{r, b-2}, l_{r, b-1}\right)$, randomly and independently generate $2^{n \hat{R}_{r}}$ sequences $\quad \hat{y}_{r, b}^{n}\left(l_{r, b} \mid m_{b}^{\prime}, m_{b-1}^{\prime}, l_{r, b-2}, l_{r, b-1}\right)$ $\prod_{i=1}^{n} P_{\hat{Y}_{r} \mid U_{r} X_{r} V_{r}}\left(\hat{y}_{r, b, i} \mid u_{r, b, i}, x_{r, b, i}, v_{r, b, i}\right)$.

- For each $d \in \mathcal{D}$ and block $b \in[1: B+$ $2]$, randomly and independently generate $2^{n \hat{R}_{d}}$ sequences $v_{d, b}^{n}\left(l_{d, b-2}\right) \sim \prod_{i=1}^{n} P_{V_{d}}\left(v_{d, b, i}\right)$, with $l_{d, b-2} \in$ $\left[1: 2^{n \hat{R}_{d}}\right]$. For each $l_{d, b-2}$, randomly and independently generate $2^{n \hat{R}_{d}}$ sequences $x_{d, b}^{n}\left(l_{d, b-1} \mid l_{d, b-2}\right) \sim$ $\prod_{i=1}^{n} P_{X_{d} \mid V_{d}}\left(x_{d, b, i} \mid v_{d, b, i}\right), l_{d, b-1} \in\left[1: 2^{n \hat{R}_{d}}\right]$. For each $l_{d, b-2}$, randomly and independently generate $2^{n R^{\prime}}$ sequences $u_{d, b}^{n}\left(m_{b}^{\prime} \mid l_{d, b-2}\right) \sim \prod_{i=1}^{n} P_{U_{d} \mid V_{d}}\left(u_{d, b, i} \mid v_{d, b, i}\right)$. For each $\left(l_{d, b-2}, l_{d, b-1}\right)$, randomly and independently generate $2^{n \hat{R}_{d}}$ sequences $\hat{y}_{d, b}^{n}\left(l_{d, b} \mid l_{d, b-2}, l_{d, b-1}\right) \sim$ $\prod_{i=1}^{n} P_{\hat{Y}_{d} \mid X_{d} V_{d}}\left(\hat{y}_{d, b, i} \mid x_{d, b, i}, v_{d, b, i}\right)$.

For each $\left(m_{b}^{\prime}, m_{b-1}^{\prime}, \mathbf{l}_{b-2}\right)$, randomly and independently generate $2^{n R^{\prime \prime}}$ sequences $x_{1, b}^{n}\left(m_{b}^{\prime \prime} \mid m_{b}^{\prime}, m_{b-1}^{\prime}, \mathbf{l}_{b-2}\right) \sim$ $\prod_{i=1}^{n} P_{X_{1} \mid U_{2}^{N} V_{2}^{N}}\left(x_{1, b, i} \mid v_{2, b, i}, u_{2, b, i}, \ldots, v_{N, b, i}, u_{N, b, i}\right)$.

Let $\mathbf{v}_{b}^{\prime n}(\mathcal{R}):=\left[v_{r, b}^{n}\left(\hat{m}_{b-1}^{\prime}, \hat{l}_{r, b-2}\right), r \in \mathcal{R}\right], \mathbf{v}_{b}^{\prime n}(\mathcal{D}):=$ $\left[v_{d, b}^{n}\left(\hat{l}_{d, b-2}\right), d \in \mathcal{D}\right], \mathbf{x}_{b}^{\prime n}(\mathcal{R}):=\left[x_{r, b}^{n}\left(\hat{l}_{r, b-1} \mid \hat{m}_{b-1}^{\prime}, \hat{l}_{r, b-2}\right):\right.$ $r \in \mathcal{R}], \mathbf{x}_{b}^{\prime n}(\mathcal{D}):=\left[x_{d, b}^{n}\left(\hat{l}_{d, b-1} \mid \hat{l}_{d, b-2}\right): d \in \mathcal{D}\right], \mathbf{u}_{b}^{\prime n}(\mathcal{R}):=$ $\left[u_{r, b}^{n}\left(\hat{m}_{b}^{\prime} \mid \hat{m}_{b-1}^{\prime}, \hat{l}_{r, b-2}: r \in \mathcal{R}\right], \mathbf{u}_{b}^{\prime n}(\mathcal{D}):=\left[u_{d, b}^{n}\left(\hat{m}_{b}^{\prime} \mid \hat{l}_{d, b-2}\right):\right.\right.$ $d \in \mathcal{D}]$ and $\hat{\mathbf{y}}_{b}^{\prime n}(\mathcal{R}):=\left[\hat{y}_{r, b}^{n}\left(\hat{l}_{r, b} \mid \hat{m}_{b}^{\prime}, \hat{m}_{b-1}^{\prime}, \hat{l}_{r, b-2}, \hat{l}_{r, b-1}\right): r \in\right.$ $\mathcal{R}], \hat{\mathbf{y}}_{b}^{\prime n}(\mathcal{D}):=\left[\hat{y}_{d, b}^{n}\left(\hat{l}_{d, b} \mid \hat{l}_{d, b-2}, \hat{l}_{d, b-1}\right): d \in \mathcal{D}\right]$.

2) Source encoding At each block $b \in[1: B+1]$, after observing $Y_{1, b}^{n}$, it looks for $\hat{\mathbf{l}}_{b-1}$ such that

$$
\begin{gathered}
\left(x_{1, b}^{n}\left(m_{b}^{\prime \prime} \mid m_{b}^{\prime}, m_{b-1}^{\prime}, \hat{\mathbf{l}}_{b-2}\right), \mathbf{v}_{b}^{\prime n}(\mathcal{R}), \mathbf{v}_{b}^{\prime n}(\mathcal{D}), \mathbf{x}_{b}^{\prime n}(\mathcal{R}), \mathbf{x}_{b}^{\prime n}(\mathcal{D}),\right. \\
\left.\mathbf{u}_{b}^{\prime n}(\mathcal{R}), \mathbf{u}_{b}^{\prime n}(\mathcal{D}), \hat{\mathbf{y}}_{b}^{\prime n}(\mathcal{R}), \hat{\mathbf{y}}_{b}^{\prime n}(\mathcal{D}), y_{1, b}^{n}\right) \in \mathcal{T}_{\epsilon}^{n}\left(P_{V_{2}^{N} X_{1}^{N} U_{2}^{N} \hat{Y}_{2}^{N} Y_{1}}\right)
\end{gathered}
$$

with $\hat{m}_{b}^{\prime \prime}=m_{b}^{\prime \prime}, \hat{m}_{b}^{\prime}=m_{b}^{\prime}$ and $\hat{m}_{b-1}^{\prime}=m_{b-1}^{\prime}$ since the transmitter knows source messages it sent.

After finding compression indices $\hat{\mathbf{I}}_{b-1}$, in block $b+1$ the transmitter sends $x_{1, b+1}^{n}\left(m_{b+1}^{\prime \prime} \mid m_{b+1}^{\prime}, m_{b}^{\prime}, \hat{\mathbf{l}}_{b-1}\right)$.

3) Relay encoding: Relay nodes perform mixed compressforward and partial decode-forward. In each block $b \in[1$ : $B+1]$, Relay $r \in \mathcal{R}$ looks for a unique index $\hat{m}_{b}^{\prime}$ such that

$$
\begin{aligned}
& \left(v_{r, b}^{n}\left(\hat{m}_{b-1}^{\prime}, l_{r, b-2}\right), x_{r, b}^{n}\left(l_{r, b-1} \mid \hat{m}_{b-1}^{\prime}, l_{r, b-2}\right),\right. \\
& \left.\quad u_{r, b}^{n}\left(\hat{m}_{b}^{\prime} \mid \hat{m}_{b-1}^{\prime}, l_{r, b-2}\right), y_{r, b}^{n}\right) \in \mathcal{T}_{\epsilon}^{n}\left(P_{X_{r} Y_{r} U_{r} V_{r}}\right) .
\end{aligned}
$$

then it compresses $y_{r, b}^{n}$ by finding a unique index $l_{r, b}$ such that

$$
\begin{aligned}
& \left(v_{r, b}^{n}, u_{r, b}^{n}, x_{r, b}^{n}, y_{r, b}^{n},\right. \\
& \left.\quad \hat{y}_{r, b}^{n}\left(l_{r, b} \mid \hat{m}_{b}^{\prime}, \hat{m}_{b-1}^{\prime}, l_{r, b-2}, l_{r, b-1}\right)\right) \in \mathcal{T}_{\epsilon}^{n}\left(P_{V_{r} U_{r} X_{r} Y_{r} \hat{Y}_{r}}\right) .
\end{aligned}
$$

In block $b+1$ it sends $x_{r, b+1}^{n}\left(l_{r, b} \mid \hat{m}_{b}^{\prime}, l_{r, b-1}\right)$.

4) Receiver encoding: Receiver $d \in \mathcal{D}$ compresses $y_{d, b}^{n}$ by finding a unique index $l_{d, b}$ such that

$$
\begin{aligned}
& \left(v_{d, b}^{n}\left(l_{d, b-2}\right), x_{d, b}^{n}\left(l_{d, b-1} \mid l_{d, b-2}\right),\right. \\
& \left.\quad \hat{y}_{d, b}\left(l_{d, b} \mid l_{d, b-2}, l_{d, b-1}\right), y_{d, b}^{n}\right) \in \mathcal{T}_{\epsilon}^{n}\left(P_{V_{d} X_{d} Y_{d} \hat{Y}_{d}}\right) .
\end{aligned}
$$

Then, in block $b+1$ it sends $x_{d, b+1}^{n}\left(l_{d, b} \mid l_{d, b-1}\right)$.

5) Decoding: Receiver $d \in \mathcal{D}$ performs backward decoding. For each block $b \in[B+2, \ldots, 1]$, it looks for $\left(\hat{m}_{b}^{\prime \prime}, \hat{m}_{b-1}^{\prime}, \hat{\mathbf{l}}_{b-2}\right)$ such that

$$
\begin{aligned}
& \left(x_{1, b}^{n}\left(\hat{m}_{b}^{\prime \prime} \mid \hat{m}_{b}^{\prime}, \hat{m}_{b-1}^{\prime}, \hat{\mathbf{l}}_{b-2}\right), \mathbf{v}_{b}^{\prime n}(\mathcal{R}), \mathbf{v}_{b}^{\prime n}(\mathcal{D}), \mathbf{x}_{b}^{n}(\mathcal{R}), \mathbf{x}_{b}^{\prime n}(\mathcal{D}),\right. \\
& \left.\mathbf{u}_{b}^{\prime n}(\mathcal{R}), \mathbf{u}_{b}^{\prime n}(\mathcal{D}), \hat{\mathbf{y}}_{b}^{\prime n}(\mathcal{R}), \hat{\mathbf{y}}_{b}^{\prime n}(\mathcal{D}), y_{d, b}^{n}\right) \in \mathcal{T}_{\epsilon}^{n}\left(P_{V_{2}^{N} X_{1}^{N} U_{2}^{N} \hat{Y}_{2}^{N} Y_{d}}\right) .
\end{aligned}
$$

6) Analysis: See [12] for details.

\section{REFERENCES}

[1] E. C. van der Meulen, "Three terminal communication channels, $A d v$. Appl. Probab., vol. 3, pp. 120-154, 1971.

[2] T. M. Cover and A. El Gamal, "Capacity theorems for the relay channel," IEEE Trans. Inf. Theory, vol. 25, no. 5, pp. 572-584, Sep. 1979.

[3] G. Kramer, M. Gastpar, and P. Gupta, "Cooperative strategies and capacity theorems for relay networks," IEEE Trans. Inf. Theory, vol. 51, no. 9, pp. 3037-3063, Sep. 2005.

[4] M. Yassaee and M. Aref, "Generalized compress-and-forward strategy for relay networks," Information Theory (ISIT), 2008 IEEE International Symposium on, Toronto, Canada, pp. 2683-2687, July 2008.

[5] S. Lim, Y-H Kim, A. El Gamal and S-Y Chung, "Noisy network coding," IEEE Trans. Inf. Theory, vol.57, no.5, pp. 3132-3152, May 2011

[6] J. Hou and G. Kramer, "Short message noisy network coding with a decode-forward option,” 2013. [Online]. Available: http://arxiv.org/abs/1304.1692/

[7] S. Lim, K. T. Kim, Y-H Kim, "Distributed decode-forward for multicast," Information Theory Workshop (ITW), 2014 IEEE, pp. 556-560, Nov. 2014

[8] S. Lim, K. T. Kim, Y-H Kim, "Distributed decode-forward for broadcast," Information Theory (ISIT), 2014 IEEE International Symposium on, pp. 636-640, June, 2014

[9] Y. Gabbai and S. I. Bross, "Achievable rates for the discrete memoryless relay channel with partial feedback configurations," IEEE Trans. Inf. Theory, vol.52, no.11, pp. 4989-5007, Nov. 2006

[10] F. M. J. Willems and E. C. van der Meulen, "Partial feedback for the discrete memoryless multiple-access channel," IEEE Trans. Inf. Theory, vol.29, no.2, pp. 287-290, Mar. 1983

[11] Y. Wu, M. Wigger, "Coding schemes for discrete memoryless broadcast channels with rate-limited feedback," Information Theory (ISIT), 2014 IEEE International Symposium on, pp.2127-2131, June 2014.

[12] Y. Wu, "Coding schemes for discrete memoryless multicast networks with rate-limited feedback," 2015. [Online]. Available on http://arxiv.org/abs/1504.04515 